\title{
Survival Benefits From Surgery for Stage IVa Head and Neck Squamous Cell Carcinoma: A Multi-institutional Analysis of 1,033 Cases
}

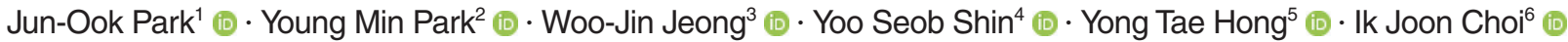

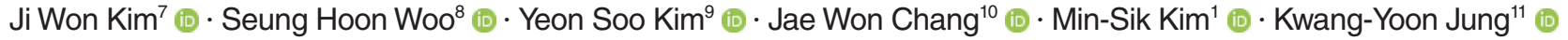

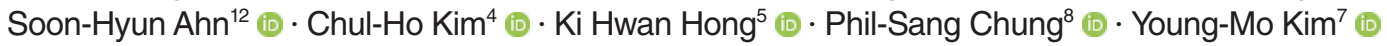 \\ Se-Heon Kim² (iD · Seung-Kuk Baek ${ }^{11}$ (i)
}

\begin{abstract}
${ }^{1}$ Department of Otolaryngology-Head and Neck Surgery, College of Medicine, The Catholic University of Korea, Seoul; ${ }^{2}$ Department of Otorhinolaryngology-Head and Neck Surgery, Yonsei University College of Medicine, Seoul; ${ }^{3}$ Department of Otorhinolaryngology-Head and Neck Surgery, Seoul National University Bundang Hospital, Seongnam; ${ }^{4}$ Department of Otolaryngology, Ajou University School of Medicine, Suwon; ${ }^{5}$ Department of Otolaryngology-Head and Neck Surgery, Jeonbuk National University Hospital, Jeonbuk National University Medical School, Jeonju; ${ }^{6}$ Department of Otorhinolaryngology-Head and Neck Surgery, Korea Cancer Center Hospital (KCCH), Korea Institute of Radiological and Medical Sciences (KIRAMS), Seoul; ${ }^{7}$ Department of Otorhinolaryngology, Inha University College of Medicine, Incheon;

${ }^{8}$ Department of Otorhinolaryngology-Head and Neck Surgery, Dankook University College of Medicine, Cheonan; ${ }^{9}$ Department of

Otorhinolaryngology, Konyang University College of Medicine, Daejeon; ${ }^{10}$ Department of Otorhinolaryngology-Head and Neck Surgery, Chungnam National University School of Medicine, Daejeon; ${ }^{11}$ Department of Otorhinolaryngology-Head and Neck Surgery, Korea University College of Medicine, Seoul; ${ }^{12}$ Department of Otorhinolaryngology-Head and Neck Surgery, Seoul National University Hospital, Seoul National University College of Medicine, Seoul, Korea
\end{abstract}

Objectives. Head and neck squamous cell carcinomas (HNSCs) are frequently diagnosed at the locoregional advanced stage (stage IVa), but controversy remains regarding whether stage IVa HSNCs should be treated with upfront surgery or definitive chemoradiation therapy (CRT). The purpose of this study was to compare overall survival (OS) and disease-free survival (DFS) in patients with stage IVa HNSC treated primarily by surgery with curative intent with/without (neo)adjuvant treatment (surgery group) versus those treated primarily with CRT (CRT group).

Methods. We reviewed data of 1,033 patients with stage IVa HNSC treated with curative intent at 17 cancer centers between 2010 and 2016.

Results. Among 1,033 patients, 765 (74.1\%) received upfront surgery and $268(25.9 \%)$ received CRT. The 5-year OS and DFS rates were $64.4 \%$ and $62.0 \%$ in the surgery group and $49.5 \%$ and $45.4 \%$ in the CRT group, respectively. In multivariate analyses, OS and DFS were better in the surgery group than in the CRT group (odds ratio [OR] for death, 0.762 ; $95 \%$ confidence interval [CI], 0.592-0.981; OR for recurrence, 0.628 ; 95\% CI, 0.492-0.802). In subgroup analyses, the OS and DFS of patients with oropharyngeal cancer were better in the surgery group (OR for death, $0.548 ; 95 \%$ CI, $0.341-0.879$; OR for recurrence, $0.598 ; 95 \%$ CI, 0.377-0.948). In the surgery group, patients with laryngeal cancer showed better OS (OR for death, $0.432 ; 95 \%$ CI, 0.211-0.882), while those with hypopharyngeal cancer DFS was improved (OR for recurrence, 0.506; 95\% CI, 0.328-0.780).

Conclusion. A survival benefit from surgery may be achieved even in patients with stage IVa HNSC, particularly those with oropharyngeal and laryngeal cancer. Surgery led to a reduction in the recurrence rate in patients with hypopharyngeal cancer.

Keywords. Squamous Cell Carcinoma of Head And Neck; Oral Neoplasms; Oropharyngeal Neoplasm; Laryngeal Neoplasms; Hypopharyngeal Neoplasms; Survival, Disease-Free Survival; Survival Rate; Prognosis; Surgery

- Received August 16, 2020

Revised September 4, 2020

Accepted September 22, 2020

- Corresponding author: Se-Heon Kim

Department of Otorhinolaryngology-Head and Neck Surgery, Yonsei

University College of Medicine, 50-1 Yonsei-ro, Seodaemun-gu,

Seoul 03722, Korea

Tel: +82-2-2228-3601, Fax: +82-2-393-0580, E-mail: shkimmd@yuhs.ac

\footnotetext{
- Co-Corresponding author: Seung-Kuk Baek

Department of Otorhinolaryngology-Head and Neck Surgery, Korea

University College of Medicine, 73 Inchon-ro, Seongbuk-gu, Seoul 02841, Korea

Tel: +82-2-920-5486, Fax: +82-2-925-5233, E-mail: mdskbaek@gmail.com
}

Copyright $(\odot) 2021$ by Korean Society of Otorhinolaryngology-Head and Neck Surgery.

This is an open-access article distributed under the terms of the Creative Commons Attribution Non-Commercial License (https://creativecommons.org/licenses/by-nc/4.0) which permits unrestricted non-commercial use, distribution, and reproduction in any medium, provided the original work is properly cited. 


\section{INTRODUCTION}

Head and neck squamous cell carcinoma (HNSC) is the sixth most common type of cancer [1], accounting for more than 700,000 new cases and 350,000 cancer deaths worldwide [2]. HNSCs may arise from the oral cavity $(354,864$ cases/yr $[2.1 \%$ of all cancers]), larynx (177,422 [1.0\%]), oropharynx $(92,887$ [0.5\%]), or hypopharynx $(80,608[0.4 \%])$ [2] and are frequently diagnosed at an advanced stage (stage IV). The prognosis of these patients is poor. Furthermore, between 2004 and 2015, the incidence rate for stage IV HNSC increased significantly, by $26.1 \%[3,4]$.

Stage IV disease is subdivided into stages IVa and IVb. In the seventh edition of the American Joint Committee on Cancer (AJCC) staging system, the term "advanced resectable" defining stage IVa was replaced with the term "moderately advanced," while the previous definition of stage IVb as "advanced unresectable" was replaced with "very advanced." A resectable tumor is one in which the gross tumor can be removed without any residual tumor, such that local control can be achieved. However, in locally advanced HNSC, identifying resectable tumors is often difficult. In addition, a substantial proportion of stage IVa disease, although resectable, is currently treated nonsurgically, either due to institutional preference or to the patient's refusal of surgery. Clear guidelines for the treatment of stage IVa disease are thus far lacking, due to the ethical concerns associated with conducting a randomized controlled trial (RCT) comparing surgery versus nonsurgical treatment in patients with advanced HNSC. Recently, several clinical trials have shown that nonsurgical treatment in some patients with advanced HNSC may lead to satisfactory oncologic outcomes [5-8]. Nonetheless, for patients with stage IVa HNSC, surgical therapy remains the preferred approach as better oncologic outcomes are expected.

To address this issue, we conducted a large-scale, multi-institutional clinical study of patients with stage IVa HNSC. Specifically, overall survival (OS) and disease-free survival (DFS) were compared in patients with stage IVa HNSC treated primarily

\section{H I G H L I G G H T S}

- It remains unclear whether upfront surgery improves overall survival (OS) and disease-free survival (DFS) in patients with stage IVa head and neck squamous cell carcinoma (HNSC) compared to definitive chemoradiation therapy.

- A survival benefit from the upfront surgical treatment may be achieved even in patients with stage IVa HNSC.

- Upfront surgical treatment improved OS and DFS in patients with stage IVa HNSC, particularly in those with oropharyngeal and laryngeal cancer.

- In patients with hypopharyngeal cancer, surgery improved DFS, but not OS. with surgery or nonsurgical modalities.

\section{MATERIALS AND METHODS}

\section{Study design}

This study was designed by the members of the Research Committee of the Korean Society of Head and Neck Surgery. The study protocol was approved by the Institutional Review Board of the Catholic University of Korea (IRB No. XC 19RCD10096) and the IRBs of all participating institutions. Data were collected retrospectively from medical reports of patients with stage IVa HNSC initially treated in 17 hospitals between January 2010 and December 2016. The hospitals belonged to the following academic medical institutions: Ajou University Hospital, Catholic Medical Center (six St. Mary's hospitals), Dankook University Hospital, Inha University Hospital, Jeonbuk National University Hospital, Korea Institute of Radiological and Medical Sciences (Korea Cancer Center Hospital), Korea University Medicine (three Korea University Hospitals), Seoul National University Bundang Hospital, and Yonsei University Health System (two Severance Hospitals). All of these institutions are high-volume centers with extensive experience in the treatment of $\mathrm{HN}$ SCs by well-trained multidisciplinary teams.

Patients who met the following criteria were included in the study: diagnosed with HNSC involving the oral cavity, oropharynx, larynx, or hypopharynx; with clinically staged Iva disease according to the 8th edition of the AJCC staging system (for human papilloma virus [HPV]-positive oropharynx cancer, stage IV disease is reserved only for distant metastases [M1 disease]. Therefore, among oropharyngeal cancer patients, only HPVnegative or not-tested patients were included in this study); no previous treatment for the same diagnosis (cancer of the head and neck) before hospitalization; no other malignant disease within 5 years at the time of diagnosis and follow up of $>12$ months. The patients were divided into two groups based on the treatment modality. Patients in the surgery group were those treated primarily by surgery with curative intent with/without (neo)adjuvant treatment, and patients in the CRT group those treated primarily by chemotherapy and radiotherapy. Clinical staging was conducted in the outpatient clinic and/or the operating room and consisted of a physical examination using a flexible or rigid endoscope and radiological evaluation such as computed tomography, magnetic resonance imaging, ultrasonography, and positron emission tomography. Therapeutic strategies differed slightly between institutions as they were decided by institutional policy, although they largely met the guideline of the National Comprehensive Cancer Network.

\section{Statistical analysis}

All analyses were performed using SPSS software ver. 16.0 (SPSS Inc., Chicago, IL, USA). A $P$-value $<0.05$ was considered to in- 
dicate statistical significance. The significance of the relationships between the two groups and clinical factors were analyzed using a chi-square test, Fisher's exact test, or Student $t$-test, as appropriate. The Kaplan Meier method was used to calculate 5-year OS and DFS rates; differences in survival rates between the two groups were assessed using the log-rank test. Multivariate analyses using a Cox proportional-hazards model were performed to compare the factors with prognostic potential indicated by univariate analyses. The primary endpoint was OS, calculated as the length of time from diagnosis until death or last follow-up. The secondary endpoint was DFS, calculated as the length of time from diagnosis until first documented recurrence or death.

\section{RESULTS}

\section{Patients}

Data were collected from 1,405 patients. After the exclusion of 372 patients who did not meet the inclusion criteria $(\mathrm{p} 16(+)$ oropharyngeal cancer [ $n=177]$, under- or over-staging [ $n=175]$, and other reasons $[\mathrm{n}=20]$ ), the final study population consisted of 1,033 patients divided among the participating institutions as follows: Ajou University Hospital ( $n=67)$, Catholic Medical Center $(n=307)$, Dankook University Hospital $(n=29)$, Inha University Hospital ( $n=51)$, Jeonbuk National University Hospital $(n=89)$, Korea Cancer Center Hospital $(n=68)$, Korea University Medicine
( $n=111)$, Seoul National University Bundang Hospital $(n=79)$ and Yonsei University Health System $(n=232)$. The schema of the cohort is described in Fig. 1. The median follow-up among surviving patients was 31 months (range, 12-132 months). The median 859 male and 174 female patients had a median age of 61 years (range, 14-101 years). Regarding clinical T stage, 150 (14.5\%), $280(27.1 \%), 152(14.7 \%)$, and $451(43.7 \%)$ patients had stage $\mathrm{T} 1$ to T4a cancers, respectively. The staging of the cervical lymph nodes was N0 in 96 patients (9.3\%), N1 in 105 patients (10.2\%), and N2 in $832(80.5 \%)$ patients. The surgery group consisted of 765 patients $(74.1 \%)$ and the CRT group contained 268 patients (25.9\%). The anatomical subsites of the HNSCs in patients of both groups are shown in Fig. 2. The baseline characteristics of the patients in the two groups are summarized in Table 1.

\section{OS of patients with HNSC}

There were 232 deaths in the surgery group and 98 in the CRT group. The 5-year OS rates were $64.4 \%$ (95\% confidence interval [CI], 62.4\%-66.4\%) and 49.5\% (95\% CI, 46.5\%-52.5\%), respectively. There were 249 and 107 overall recurrences, with 5-year DFS rates of $62.0 \%$ (95\% CI, 59.9\%-64.1\%) and $45.4 \%$ (95\% CI, $41.5 \%-49.3 \%$ ), respectively. The Kaplan-Meier survival curves are shown in Fig. 3. The results of the univariate analyses are presented in Supplementary Table 1. In multivariate analyses, OS and DFS were significantly higher in the surgery group (Table 2). The recurrence patterns in both groups are summarized

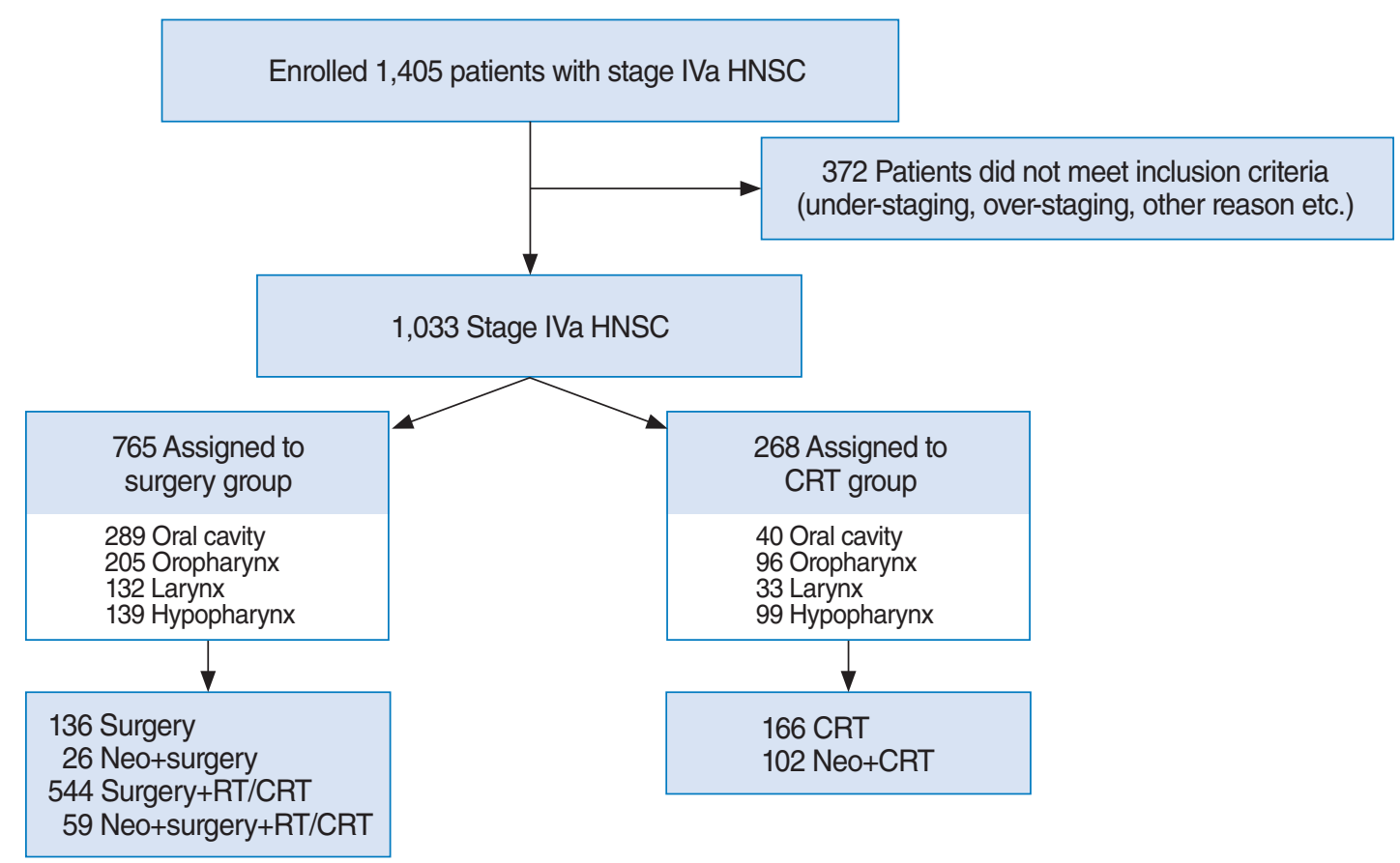

Fig. 1. Schema of the cohort. The study design, including the allocation of patients to the surgery group and CRT group according to the primary treatment modality, is shown. HNSC, head and neck squamous cell carcinoma; Surgery group, patients treated primarily by surgery with curative intent with/without (neo)adjuvant treatment; CRT group, patients treated primarily with chemotherapy and radiotherapy; Neo, neoadjuvant chemotherapy; $\mathrm{RT}$, radiotherapy; CRT, chemoradiation therapy. 


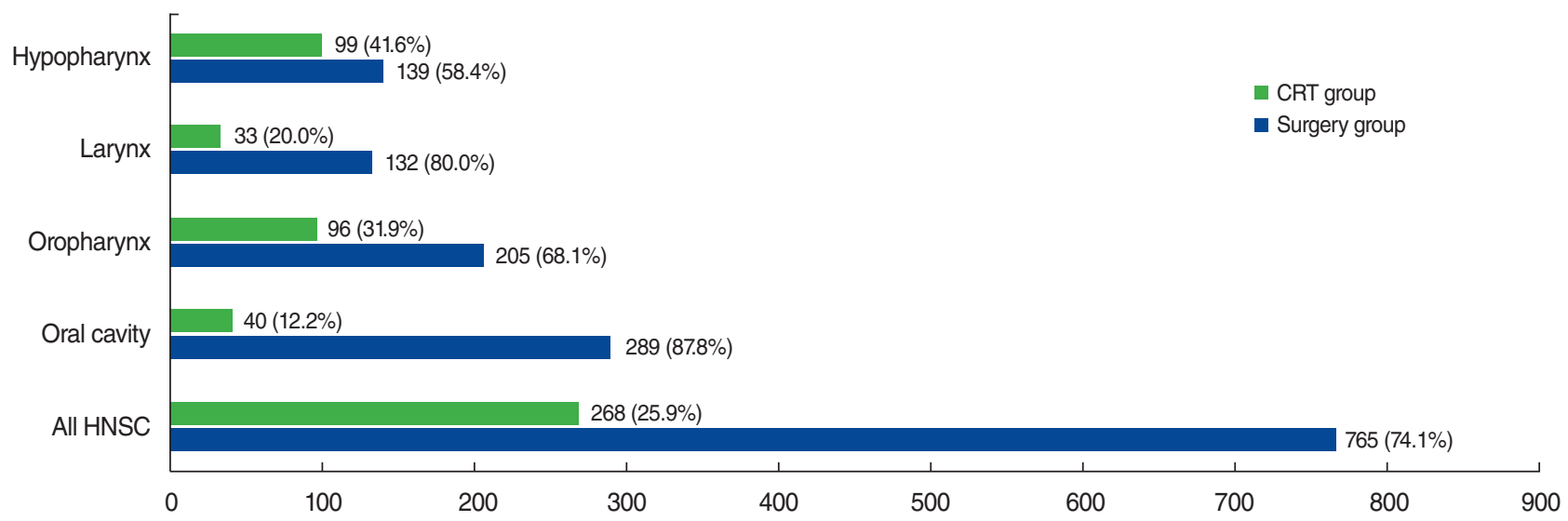

Fig. 2. The proportion of surgery-based treatments in patients $(n=1,033)$ with stage IVa head neck cancer, shown by anatomical location of the primary tumor. CRT group, patients treated primarily with chemotherapy and radiotherapy; Surgery group, patients treated primarily by surgery with curative intent with/without (neo)adjuvant treatment; HNSC, head and neck squamous cell carcinoma.

Table 1. Baseline characteristics of all patients

\begin{tabular}{|c|c|c|c|c|}
\hline Variable & Total $(n=1,033)$ & Surgery group $(n=765)$ & CRT group $(n=268)$ & $P$-value \\
\hline Age (yr) & & & & 0.979 \\
\hline$\geq 60$ & $431(41.7 / 100)$ & $319(41.7 / 74.0)$ & $112(41.8 / 26.0)$ & \\
\hline$<60$ & $602(58.3 / 100)$ & $446(58.3 / 74.1)$ & $156(58.2 / 25.9)$ & \\
\hline Sex & & & & $0.007^{*}$ \\
\hline Male & $859(83.2 / 100)$ & $622(81.3 / 72.4)$ & $237(88.4 / 27.6)$ & \\
\hline Female & $174(16.8 / 100)$ & $143(18.7 / 82.2)$ & $31(11.6 / 17.8)$ & \\
\hline T stage & & & & 0.116 \\
\hline Non T4a & $582(56.3 / 100)$ & $442(57.8 / 75.9)$ & $140(52.2 / 24.1)$ & \\
\hline $\mathrm{T} 4 \mathrm{a}$ & $451(43.7 / 100)$ & $323(42.2 / 71.6)$ & $128(47.8 / 28.4)$ & \\
\hline N stage & & & & 0.056 \\
\hline No-1 & $201(19.5 / 100)$ & $160(20.9 / 79.6)$ & $41(15.3 / 20.4)$ & \\
\hline N2 & $832(80.5 / 100)$ & $605(79.1 / 72.7)$ & $227(84.7 / 27.3)$ & \\
\hline Follow-up period (mo) & $31(12-132)$ & $33(12-122)$ & $23(12-132)$ & $0.004^{*}$ \\
\hline
\end{tabular}

Values are presented as number of patients (\%within group/\%within variable) or median (range).

Surgery group, patients treated primarily by surgery with curative intent with/without (neo)adjuvant treatment; CRT group, patients treated primarily with chemotherapy and radiotherapy.

*Statistically significant $(P<0.05)$.
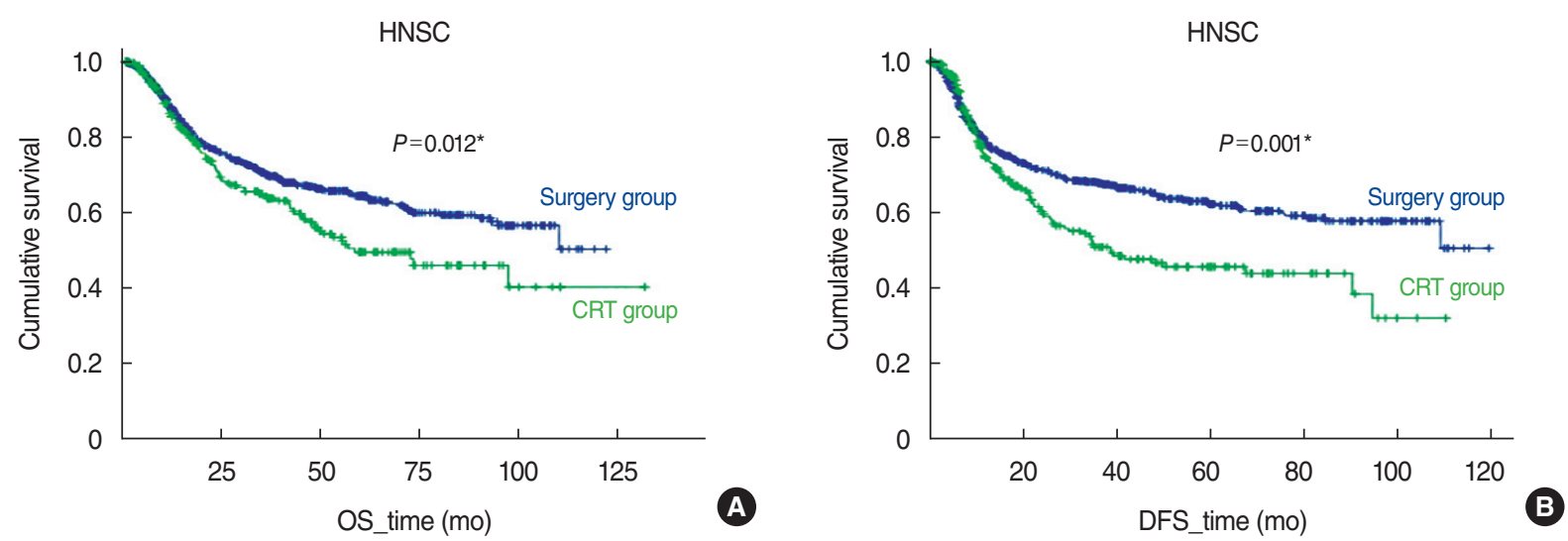

Fig. 3. Kaplan-Meier survival curves for patients with stage IVa HNSC ( $n=1,033)$. (A) Overall survival (OS), (B) disease-free survival (DFS) treated surgically (surgery group) or nonsurgically (CRT group). HNSC, head and neck squamous cell carcinoma; Surgery group, patients treated primarily by surgery with curative intent with/without (neo)adjuvant treatment; CRT group, patients treated primarily with chemotherapy and radiotherapy. ${ }^{*}$ Statistically significant $(P<0.05)$. 
in Supplementary Table 2. The majority of first recurrences were locoregional in both groups. There were 163 recurrences in 765 patients $(21.2 \%)$ in the surgery group, and 94 recurrences in 268 patients $(34.9 \%)$ in the CRT group.

\section{Survival according to HNSC subgroups}

The benefits of surgery as determined by OS and DFS were evaluated according to the prognostic indicators identified in the univariate analyses.

\section{Primary tumor site}

The Kaplan-Meier survival curves based on the primary tumor site are shown in Supplementary Fig. 1 and Fig. 4. In the multivariate analyses, OS and DFS rates were significantly higher in patients with oropharyngeal and laryngeal cancer than in those with oral cancer. The results are summarized in Table 2.

\section{1) Oral cavity}

In the surgery group, the 5-year OS and DFS rates were $59.8 \%$ (95\% CI, 56.4\%-63.2\%) and 52.5\% (95\% CI, 49.0\%-56.0\%), respectively. In the CRT group the corresponding rates were
53.1\% (95\% CI, 42.4\%-63.8\%) and 39.3\% (95\% CI, 26.9\%$51.7 \%$ ), respectively. The differences between the two groups were not significant.

\section{2) Oropharynx}

The 5-year OS and DFS rates were 76.9\% (95\% CI, 73.5\%$80.3 \%$ ) and $71.8 \%$ (95\% CI, $68.2 \%-75.4 \%$ ), respectively, in the surgery group and $53.1 \%(95 \% \mathrm{CI}, 46.4 \%-59.8 \%)$ and $54.5 \%$ (95\% CI, $48.2 \%-60.8 \%$ ), respectively, in the CRT group. In the multivariate analyses, after adjustment for covariates, OS and DFS were significantly higher in the surgery group (Supplementary Table 3, Fig. 5).

\section{3) Larynx}

The OS and DFS rates were 69.0\% (95\% CI, 64.0\%-74.0\%) and $63.9 \%(95 \% \mathrm{CI}, 58.1 \%-69.7 \%)$, and $42.9 \%(95 \% \mathrm{CI}$, $29.6 \%-56.2 \%)$ and $53.4 \%(95 \%$ CI, 41.8\%-65.0\%), respectively, in the two groups. In multivariate analyses, after adjustment for covariates, OS was significantly higher in the surgery group (Supplementary Table 4, Fig. 5). Patients in the surgery group included those who underwent total laryngectomy $(n=84$,

Table 2. Multivariate analyses (Cox proportional hazard model) of the clinical parameters predicting the prognosis of patients with stage IVa $\operatorname{HNSC}(n=1,033)$

\begin{tabular}{|c|c|c|c|c|}
\hline \multirow{2}{*}{ Variable } & \multicolumn{2}{|c|}{ Overall survival } & \multicolumn{2}{|c|}{ Disease-free survival } \\
\hline & OR $(95 \% \mathrm{Cl})$ & $P$-value & OR $(95 \% \mathrm{Cl})$ & $P$-value \\
\hline Age $\geq 60 \mathrm{yr}$ & $1.550(1.231-1.952)$ & $0.001^{*}$ & $1.386(1.112-1.728)$ & $0.004^{*}$ \\
\hline Primary site & & $<0.001^{*}$ & & $<0.001^{*}$ \\
\hline Oral cavity & 1.000 & - & 1.000 & - \\
\hline Oropharynx & $0.558(0.410-0.757)$ & $<0.001^{*}$ & $0.522(0.390-0.698)$ & $<0.001^{*}$ \\
\hline Larynx & $0.615(0.429-0.881)$ & $0.008^{*}$ & $0.631(0.454-0.878)$ & $0.006^{*}$ \\
\hline Hypopharynx & $1.031(0.771-1.379)$ & 0.835 & $0.747(0.558-1.000)$ & 0.050 \\
\hline T4a stage & $1.157(0.924-1.450)$ & 0.204 & $1.362(1.094-1.697)$ & $0.006^{*}$ \\
\hline Surgery group & $0.762(0.592-0.981)$ & $0.035^{*}$ & $0.628(0.492-0.802)$ & $<0.001^{*}$ \\
\hline
\end{tabular}

HNSC, head and neck squamous cell carcinoma; OR, odds ratio; Cl, confidence interval; Surgery group, patients treated primarily by surgery with curative intent with/without (neo)adjuvant treatment.

*Statistically significant $(P<0.05)$.
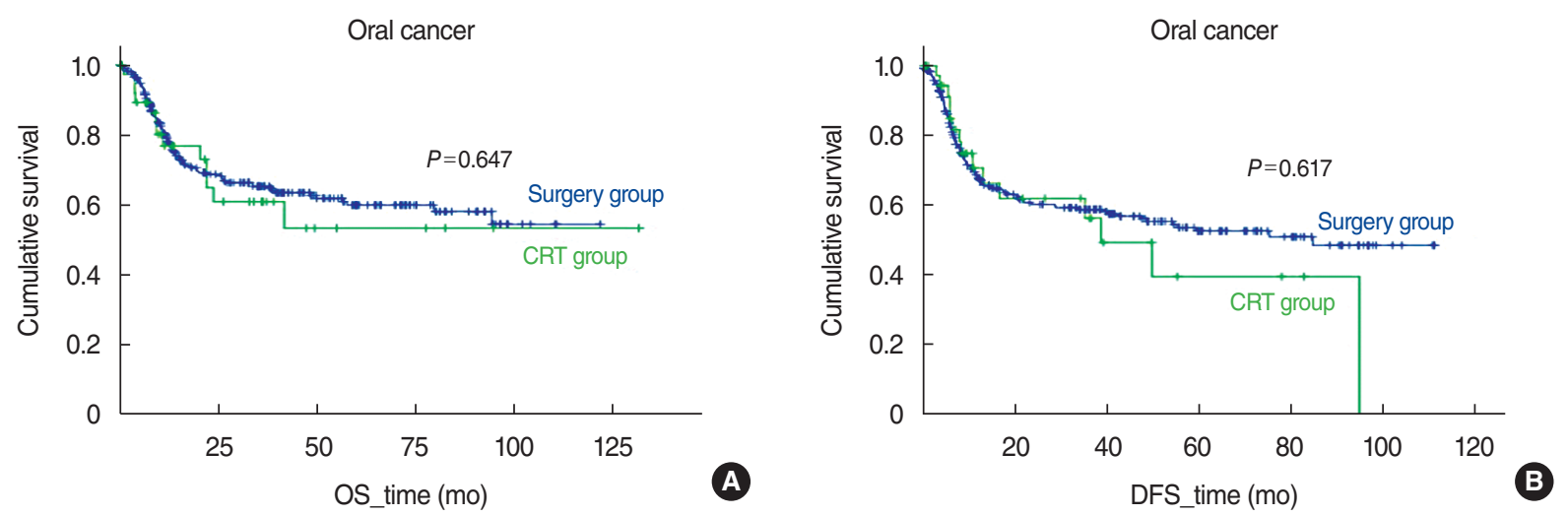

Fig. 4. Kaplan-Meier survival curves according to treatment modality and primary tumor site or T4a stage. Oral cancer $(n=329)$ : $(A)$ overall survival (OS) and (B) disease-free survival (DFS). 

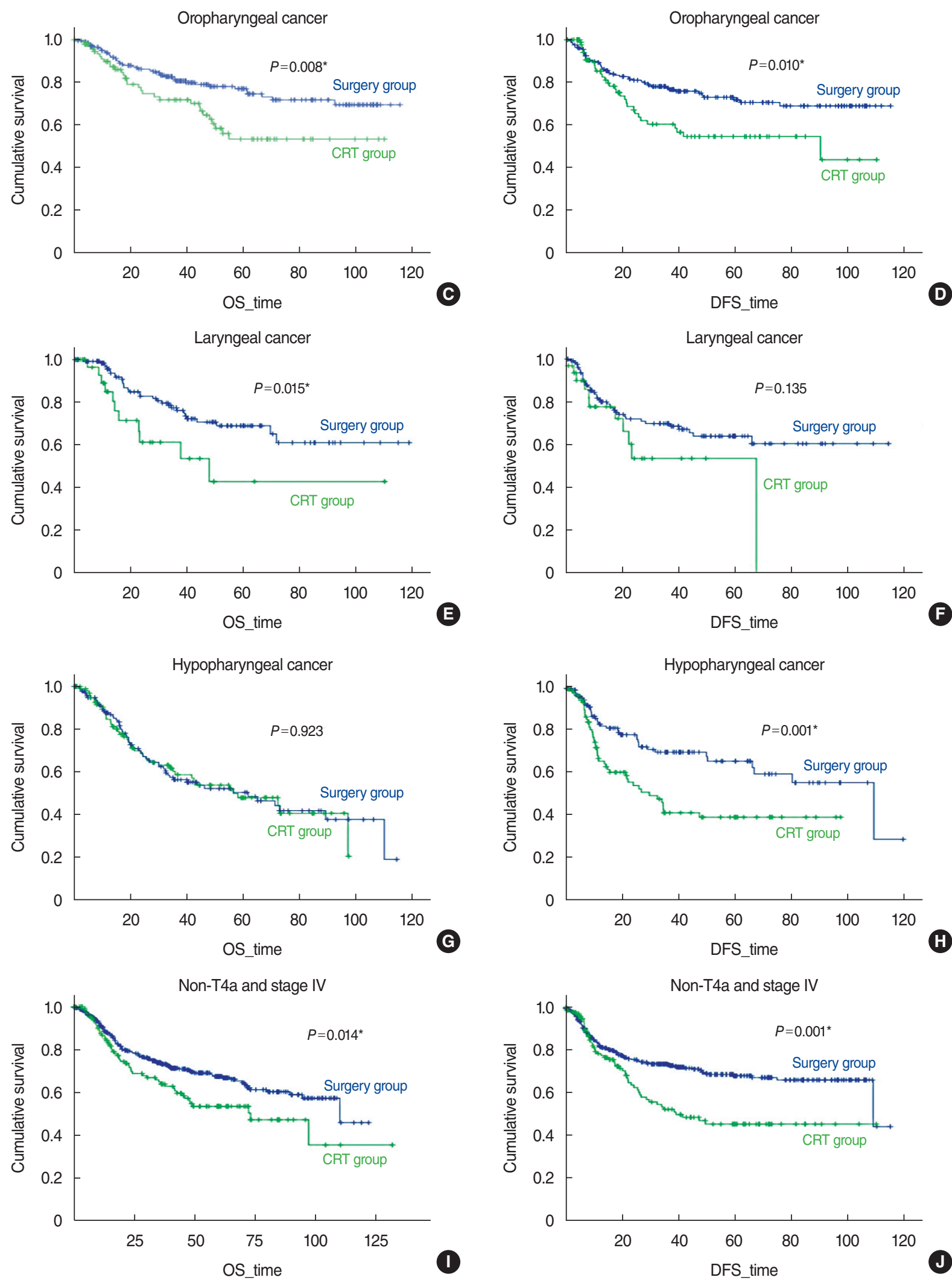

Fig. 4. (Continued) Oropharynx ( $n=301)$ : (C) OS and (D) DFS. Larynx ( $n=165)$ : (E) OS and (F) DFS. Hypopharynx ( $n=238)$ : (G) OS and (H) DFS. Non-T4a ( $n=582$ ): (I) OS and (J) DFS. Surgery group, patients treated primarily by surgery with curative intent with/without (neo)adjuvant treatment; CRT group, patients treated primarily with chemotherapy and radiotherapy. *Statistically significant $(P<0.05)$. 


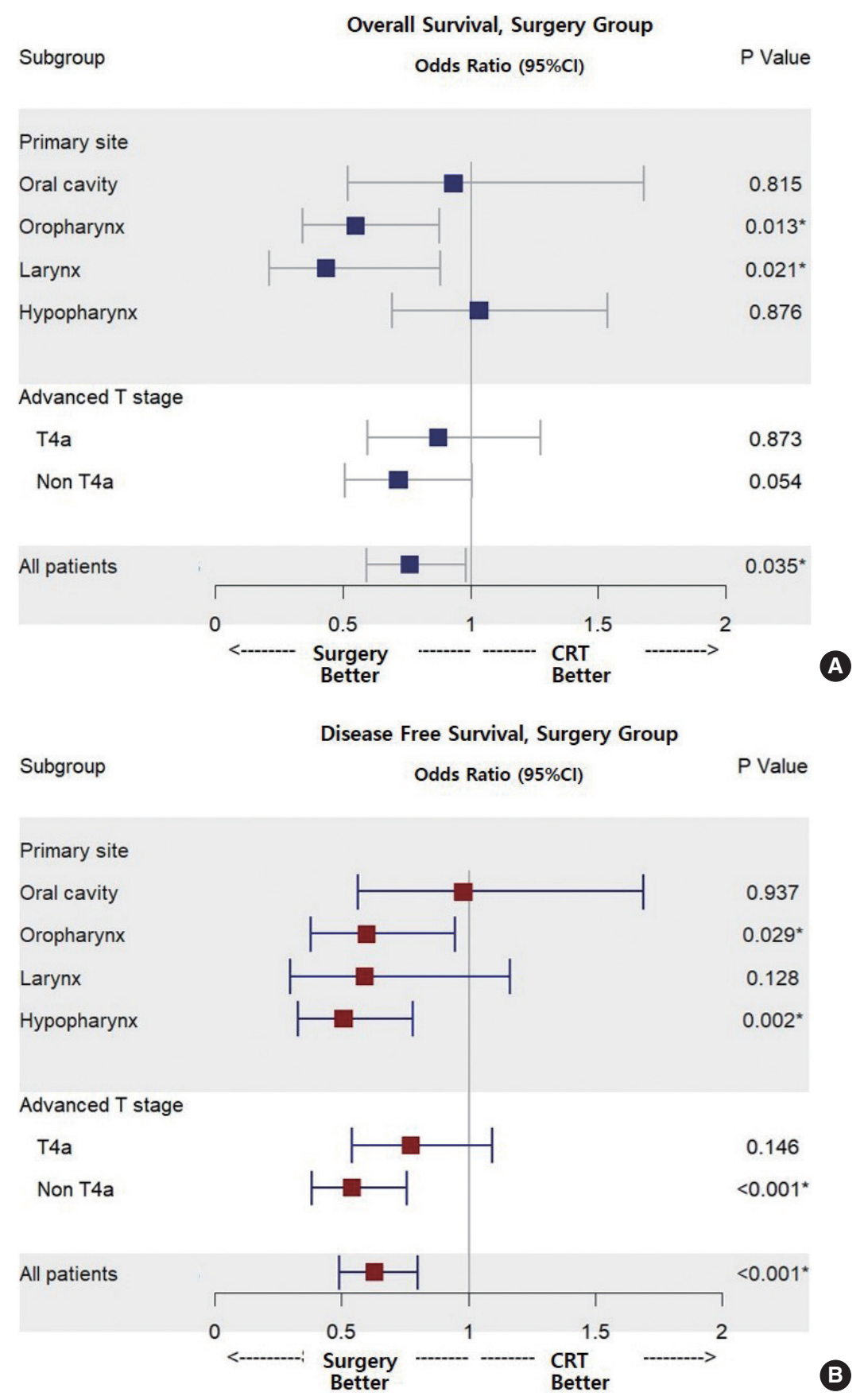

Fig. 5. Survival benefits from surgery by primary tumor site or T4a stage. (A) Overall survival, (B) disease-free survival. Cl, confidence interval; CRT group, patients treated primarily with chemotherapy and radiotherapy. ${ }^{\star}$ Statistically significant $(P<0.05)$.

$63.6 \%)$ and those who underwent partial laryngectomy $(n=48$, $36.4 \%)$.

\section{4) Hypopharynx}

The OS and DFS rates were 50.2\% (95\% CI, 45.1\%-52.3\%) and $64.5 \%$ (95\% CI, 59.2\%-69.8\%), and $47.7 \%$ (95\% CI, $41.6 \%-53.8 \%$ ) and $37.7 \%$ (95\% CI, 31.8\%-43.6\%), respectively. In multivariate analyses, after adjustment for covariates,
DFS was significantly higher in the surgery group (Supplementary Table 5, Fig. 5).

T4a vs. non-T4a disease

1) T4a disease

For patients with stage T4a disease, the 5-year OS and DFS rates were $59.9 \%$ (95\% CI, 56.6\%-63.2\%) and 53.5\% (95\% CI, $50.1 \%-56.9 \%$ ), respectively, in the surgery group and $42.2 \%$ 
(95\% CI, 35.3\%-49.1\%) and 46.6\% (95\% CI, 40.8\%-52.4\%), respectively, in the CRT group. The differences between the two groups were not significant $(P=0.340$ and $P=0.234)$.

\section{2) Non-T4a disease (T1-3N2)}

For patients with non-T4a stage disease, the OS and DFS rates were $67.4 \%(95 \% \mathrm{CI}, 64.8 \%-70.0 \%)$ and $67.8 \%(95 \% \mathrm{CI}$, $65.2 \%-70.4 \%$ ), and $53.4 \%$ (95\% CI, $48.4 \%-58.4 \%$ ) and $45.1 \%$ (95\% CI, 39.8\%-50.4\%), respectively. The differences between the two groups were significant $(P=0.014$ and $P=0.001)$. In multivariate analyses, OS was higher in the surgery group (OR for death, 0.716 ; $95 \%$ CI, 0.509-1.006) but did not significantly differ from that of the CRT group $(P=0.054)$. By contrast, DFS was significantly higher in the surgery group (OR for recurrence, 0.538 ; 95\% CI, 0.382-0.759; $P<0.001$ ) (Supplementary Table 6, Fig. 5).

\section{DISCUSSION}

To the best of our knowledge, this is the largest study of a stage IVa HNSC cohort reported in the English-language medical literature. Ethical issues have hindered RCTs comparing the oncologic outcomes of advanced HNSC patients treated surgically or nonsurgically. In major hospitals of South Korea, $74.1 \%$ of patients with advanced HNSC have been treated surgically during the past 6 years (2010-2016), a therapeutic strategy supported by our results showing improved survival in these patients. After adjustment for covariates expected to affect prognosis, surgery reduced the odds of death and disease recurrence in patients with stage IVa HNSC by $26 \%$ and $38 \%$, respectively. In their RCT, Iyer et al. [9] found either no differences in outcomes or a slight advantage favoring surgery-based treatment in stage III/IV HNSC. However, that study included a broad spectrum of cancer stages, from T1N2M0 to T4N2M0, and did not specifically address the prognosis of patients with stage IVa disease. An additional benefit of surgery is that it generates pathologic information, including surgical margins, perivascular invasion, perineural invasion, and pathologic stage, all of which are potent prognostic predictors and may lead to patient-tailored treatment, including the need for chemotherapy or radiation therapy. In our patient cohort, $80 \%$ of patients in the surgery group underwent adjuvant treatment due to positive surgical margins $(30 \%)$ or other reasons $(50 \%)$.

Surgery is the generally accepted treatment of choice for oral cancer.Thus, analyses of survival in patients treated surgically or nonsurgically were not possible, as the majority of patients were treated by surgery and a control group of chemoradiation therapy (CRT) patients was not available. Previous reports also support surgery as the preferred treatment in oral cancer [9-11]. In the RCT of Iyer et al. [9], among 32 patients with locally advanced oral cancer, those who underwent surgery followed by radiotherapy (SRT) had better survival than those treated with
CRT (68\% vs. $12 \%$ ). Spiotto et al. [10] used the National Cancer Database to compare the survival outcomes of 6,900 patients with stage III/IVa oral cancer. SRT was associated with better survival than CRT alone (3-year OS, $53.9 \%$ vs. $37.8 \%$ ).

Among oropharyngeal cancers, the incidence of HPV-positive tumors is steadily increasing but their characteristics differ from those of HPV-negative tumors $[12,13]$. In our study, only HPVnegative or non-tested patients were included. In these patients, surgery reduced the odds of death by $46 \%$ and disease recurrence by $41 \%$. Our findings suggest that surgery-based treatments can achieve better survival than CRT in patients with HPV-negative oropharyngeal cancer. However, the results reported in the literature vary. The multicenter retrospective study by Song et al. [14] compared the prognosis of 586 patients treated by SRT versus CRT, including 419 patients with stage IVa oropharyngeal cancer, and reported similar survival outcomes. Zenga et al. [15] reported that among patients with $\mathrm{T} 4$ oropharyngeal cancer, particularly in the era of HPV-positive disease, nonsurgical treatment had a significant negative association with OS (OR, 2.79), disease-specific survival (OR, 3.38), and DFS (OR, 2.59). Kelly et al. [16] compared survival outcomes among patients with cT1-2 N1-2b HPV-negative oropharyngeal cancer and found that upfront surgery was not associated with a better OS than achieved with CRT (OR, 1.01). Roden et al. [17] analyzed the National Cancer Database and found that although CRT was the most common treatment modality for stage III-IVb oropharyngeal cancer $(48.1 \%)$ in the United States, patients who underwent surgery and CRT had a higher 3-year OS (88.5\%) than did patients treated with CRT alone (74.2\%).

Surgery reduced the odds of overall death by $57 \%$ in our patients with laryngeal cancer. However, two randomized trials supporting nonsurgical treatment have had a significant impact on actual practice $[18,19]$. Trends in the treatment of advanced laryngeal cancer in the United States [20] changed between 1985 and 2007, with CRT increasing from $7 \%$ to $45 \%$ and surgery decreasing from $42 \%$ to $32 \%$. However, these two trials covered stage III/IV disease (possibly analyzing T1N2M0 and T4N2M0 together), which may have resulted in biased results. A study based on the data of 7,019 patients from a national hospital-based cancer registry [21] reported that treatment outcomes for patients with stage III and IV disease differed, thus complicating drawing a unique conclusion. A study by Patel et al. [22] using data from 8,703 patients entered in the National Cancer Database showed that for those with T4 disease, surgery improved OS (OR, 0.80; $95 \% \mathrm{CI}, 0.62-0.92)$, but in patients with non-T4 disease and a high nodal burden (T2-T3N2-N3) it worsened survival (OR, 1.25; 95\% CI, 1.04-1.51) compared to CRT. Other studies [23,24] have also reported better survival in patients with $\mathrm{T} 4$ disease treated surgically than nonsurgically and recommended total laryngectomy for those with T4 laryngeal cancer.

Whether patients with advanced hypopharyngeal cancer without distant metastasis should be treated by SRT or CRT remains 
controversial. Retrospective studies have included patients with stage III and IV disease and the results have differed. To the best of our knowledge, ours is the first study limited to stage IVa hypopharyngeal cancer in a large patient population. Although surgery did not confer a survival benefit, it reduced the odds of recurrence by $49 \%$. Chung et al. [25] reported very similar results for 266 patients with stage III/IV disease. They showed that survival following nonsurgical treatment $(44.6 \%$ for induction chemotherapy followed by [chemo]radiotherapy [ICT], 39.6\% for CRT) was comparable to that of SRT (45.3\%) and that the salvage rate after nonsurgical treatment was higher $(12.5 \%$ for ICT, $15.6 \%$ for CRT, and 3.8\% for SRT). Some authors have reported that survival and recurrence rates are not significantly different between treatment modalities [26,27], whereas others have reported that surgery improves OS and DFS in patients with stage III/IV disease [28].

The main limitation of our study was its retrospective design. In addition, as this was a multi-center study, patients were inevitably treated using slightly different modalities. In addition, the number of patients in the CRT group was relatively small, particularly in the subgroup with oral cancer, which is almost always treated surgically. Despite these limitations, our study provides the basis for a much-needed guideline for the treatment of stage IVa HNSC.

In summary, surgery can be expected to confer survival benefits in patients with stage IVa HNSC and OS was improved in patients with stage IVa oropharyngeal and laryngeal cancer. Furthermore, surgery reduced the recurrence rate in patients with hypopharyngeal cancer, although it did not improve OS. However, overall, surgery does not confer a survival benefit in patients with advanced T stage (T4a) HNSCs.

\section{CONFLICT OF INTEREST}

No potential conflict of interest relevant to this article was reported.

\section{ACKNOWLEDGMENTS}

This study was conducted by the members of the Research Committee of the Korean Society of Head and Neck Surgery.

\section{ORCID}

Jun-Ook Park Young Min Park Woo-Jin Jeong Yoo Seob Shin Yong Tae Hong
Ik Joon Choi JiWon Kim Seung Hoon Woo Yeon Soo Kim Jae Won Chang Min-Sik Kim Kwang-Yoon Jung Soon-Hyun Ahn Chul-Ho Kim Ki Hwan Hong Phil-Sang Chung Young-Mo Kim Se-Heon Kim Seung-Kuk Baek https://orcid.org/0000-0002-9680-3873 https://orcid.org/0000-0003-1587-9671 https://orcid.org/0000-0001-7560-1140 https://orcid.org/0000-0002-7862-1662 https://orcid.org/0000-0002-6596-931X https://orcid.org/0000-0001-6222-8359 https://orcid.org/0000-0003-4316-0779 https://orcid.org/0000-0002-0759-6850 https://orcid.org/0000-0002-2161-4488 https://orcid.org/0000-0003-2192-4838 https://orcid.org/0000-0003-4591-2276 https://orcid.org/0000-0003-0016-0904 https://orcid.org/0000-0002-6407-5859 https://orcid.org/0000-0002-4751-0337

\section{AUTHOR CONTRIBUTIONS}

Conceptualization: JOP, SKB. Data curation: all authors. Formal analysis: JOP, SKB. Methodology: JOP, YMP,WJJ, YSS, YTH, IJC, JWK, SHW, YSK, JWC, SKB. Project administration: SHK, SKB. Visualization: JOP. Writing-original draft: JOP. Writing-review \& editing: JOP, SKB.

\section{SUPPLEMENTARY MATERIALS}

Supplementary materials can be found via https://doi.org/10. 21053/ceo.2020.01732.

\section{REFERENCES}

1. Vigneswaran N,Williams MD. Epidemiologic trends in head and neck cancer and aids in diagnosis. Oral Maxillofac Surg Clin North Am. 2014 May;26(2):123-41.

2. Bray F, Ferlay J, Soerjomataram I, Siegel RL, Torre LA, Jemal A. Global cancer statistics 2018: GLOBOCAN estimates of incidence and mortality worldwide for 36 cancers in 185 countries. CA Cancer J Clin. 2018 Nov;68(6):394-424.

3. Thompson-Harvey A, Yetukuri M, Hansen AR, Simpson MC, Adjei Boakye E, Varvares MA, et al. Rising incidence of late-stage head and neck cancer in the United States. Cancer. 2020 Mar;126(5): 1090-101.

4. Gatta G, Botta L, Sanchez MJ,Anderson LA, Pierannunzio D, Licitra $\mathrm{L}$, et al. Prognoses and improvement for head and neck cancers diagnosed in Europe in early 2000s: The EUROCARE-5 populationbased study. Eur J Cancer. 2015 Oct;51(15):2130-43.

5. Denis F, Garaud P, Bardet E, Alfonsi M, Sire C, Germain T, et al. Final results of the 94-01 French Head and Neck Oncology and Radiotherapy Group randomized trial comparing radiotherapy alone with concomitant radiochemotherapy in advanced-stage oropharynx carcinoma. J Clin Oncol. 2004 Jan;22(1):69-76.

6. Lefebvre JL, Chevalier D, Luboinski B, Kirkpatrick A, Collette L, Sahmoud T. Larynx preservation in pyriform sinus cancer: preliminary results of a European Organization for Research and Treatment of 
Cancer phase III trial. EORTC Head and Neck Cancer Cooperative Group. J Natl Cancer Inst. 1996 Jul;88(13):890-9.

7. Forastiere AA, Zhang Q,Weber RS, Maor MH, Goepfert H, PajakTF, et al. Long-term results of RTOG 91-11: a comparison of three nonsurgical treatment strategies to preserve the larynx in patients with locally advanced larynx cancer. J Clin Oncol. 2013 Mar;31(7):845-52.

8. Chaukar DA,Walvekar RR, Das AK, Deshpande MS, Pai PS, Chaturvedi P, et al. Quality of life in head and neck cancer survivors: a crosssectional survey. Am J Otolaryngol. 2009 May-Jun;30(3):176-80.

9. Iyer NG, Tan DS, Tan VK, Wang W, Hwang J, Tan NC, et al. Randomized trial comparing surgery and adjuvant radiotherapy versus concurrent chemoradiotherapy in patients with advanced, nonmetastatic squamous cell carcinoma of the head and neck: 10-year update and subset analysis. Cancer. 2015 May;121(10):1599-607.

10. Spiotto MT, Jefferson G, Wenig B, Markiewicz M,Weichselbaum RR, Koshy M. Differences in survival with surgery and postoperative radiotherapy compared with definitive chemoradiotherapy for oral cavity cancer: a national cancer database analysis. JAMA Otolaryngol Head Neck Surg. 2017 Jul;143(7):691-9.

11. Gore SM, Crombie AK, Batstone MD, Clark JR. Concurrent chemoradiotherapy compared with surgery and adjuvant radiotherapy for oral cavity squamous cell carcinoma. Head Neck. 2015 Apr;37(4): 518-23.

12. D’Souza G, Kreimer AR, Viscidi R, Pawlita M, Fakhry C, Koch WM, et al. Case-control study of human papillomavirus and oropharyngeal cancer. N Engl J Med. 2007 May;356(19):1944-56.

13. Keane FK, Chen YH, Neville BA, Tishler RB, Schoenfeld JD, Catalano $\mathrm{PJ}$, et al. Changing prognostic significance of tumor stage and nodal stage in patients with squamous cell carcinoma of the oropharynx in the human papillomavirus era. Cancer. 2015 Aug;121(15): 2594-602.

14. Song S, Wu HG, Lee CG, Keum KC, Kim MS, Ahn YC, et al. Chemoradiotherapy versus surgery followed by postoperative radiotherapy in tonsil cancer: Korean Radiation Oncology Group (KROG) study. BMC Cancer. 2017 Aug;17(1):598.

15. Zenga J,Wilson M, Adkins DR, Gay HA, Haughey BH, Kallogjeri D, et al. Treatment outcomes for T4 oropharyngeal squamous cell carcinoma. JAMA Otolaryngol Head Neck Surg. 2015 Dec;141(12): 1118-27.

16. Kelly JR, Park HS, An Y, Contessa JN, Yarbrough WG, Burtness BA, et al. Comparison of survival outcomes among human papillomavirus-negative cT1-2 N1-2b patients with oropharyngeal squamous cell cancer treated with upfront surgery vs definitive chemoradiation therapy: an observational study. JAMA Oncol. 2017 Aug;3(8):1107-11.

17. Roden DF, Schreiber D, Givi B. Triple-modality treatment in patients with advanced stage tonsil cancer. Cancer. 2017 Sep;123(17): 3269-76.
18. Department of Veterans Affairs Laryngeal Cancer Study Group,Wolf GT, Fisher SG, Hong WK, Hillman R, Spaulding M, et al. Induction chemotherapy plus radiation compared with surgery plus radiation in patients with advanced laryngeal cancer. N Engl J Med. 1991 Jun;324(24):1685-90.

19. Forastiere AA, Goepfert H, Maor M, PajakTF, Weber R, Morrison W, et al. Concurrent chemotherapy and radiotherapy for organ preservation in advanced laryngeal cancer. N Engl J Med. 2003 Nov;349(22): 2091-8.

20. Chen AY, Fedewa S, Zhu J.Temporal trends in the treatment of earlyand advanced-stage laryngeal cancer in the United States, 1985-2007. Arch Otolaryngol Head Neck Surg. 2011 Oct;137(10):1017-24.

21. Chen AY, Halpern M. Factors predictive of survival in advanced laryngeal cancer. Arch Otolaryngol Head Neck Surg. 2007 Dec;133(12): 1270-6.

22. Patel SA, Qureshi MM, Dyer MA, Jalisi S, Grillone G, Truong MT. Comparing surgical and nonsurgical larynx-preserving treatments with total laryngectomy for locally advanced laryngeal cancer. Cancer. 2019 Oct;125(19):3367-77.

23. Dziegielewski PT, O’Connell DA, Klein M, Fung C, Singh P, Alex Mlynarek M, et al. Primary total laryngectomy versus organ preservation for T3/T4a laryngeal cancer: a population-based analysis of survival. J Otolaryngol Head Neck Surg. 2012 Apr;41 Suppl 1:S56-64.

24. Tang ZX, Gong JL, Wang YH, Li ZH, He Y, Liu YX, et al. Efficacy comparison between primary total laryngectomy and nonsurgical organ-preservation strategies in treatment of advanced stage laryngeal cancer: a meta-analysis. Medicine (Baltimore). 2018 May;97(21): e10625.

25. Chung EJ, Jeong WJ, Jung YH, Kwon SK, Kwon TK, Ahn SH, et al. Long-term oncological and functional outcomes of induction chemotherapy followed by (chemo)radiotherapy vs definitive chemoradiotherapy vs surgery-based therapy in locally advanced stage III/IV hypopharyngeal cancer: multicenter review of 266 cases. Oral Oncol. 2019 Feb;89:84-94.

26. Kim JW, Kim MS, Kim SH, Kim JH, Lee CG, Kim GE, et al. Definitive chemoradiotherapy versus surgery followed by adjuvant radiotherapy in resectable stage III/IV hypopharyngeal cancer. Cancer ResTreat. 2016 Jan;48(1):45-53.

27. Iwae S, Fujii M, Hayashi R, HasegawaY, FujiiT, Okami K, et al. Matchedpair analysis of patients with advanced hypopharyngeal cancer: surgery versus concomitant chemoradiotherapy. Int J Clin Oncol. 2017; 22(6):1001-8.

28. Vengaloor Thomas T, Nittala MR, Bhanat E, Albert AA, Vijayakumar S. Management of advanced-stage hypopharyngeal carcinoma: 25year experience from a tertiary care medical center. Cureus. 2020 Jan;12(1):e6679. 